\title{
The current role and future prospectives of functional parameters by diffusion weighted imaging in the assessment of histologic grade of HCC
}

\author{
Vincenza Granata ${ }^{1}$, Roberta Fusco ${ }^{1,2^{*}}$, Salvatore Filice ${ }^{1}$, Orlando Catalano ${ }^{1}$, Mauro Piccirillo ${ }^{2}$, Raffaele Palaia ${ }^{2}$,
} Francesco Izzo ${ }^{2}$ and Antonella Petrillo ${ }^{1}$

\begin{abstract}
Hepatocellular carcinoma (HCC) is one of the most common human solid malignancies worldwide. Although the MRI is the technique that is best adapted to characterize HCC, there is not an agreement regarding the study protocol and even what the role of Diffusion-weighted imaging (DWI). The possibility that imaging study can correlate to histologic grade to selecting the therapeutic strategy would be valuable in helping to direct the proper management of HCC. Apparent Diffusion Coefficient (ADC) and IVIM-derived perfusion fraction (fp) and tissue diffusivity (Dt) values of HCC showed significantly better diagnostic performance in differentiating high-grade HCC from low-grade HCC, and significant correlation was observed between ADC, fp, Dt and histological grade.
\end{abstract}

Keywords: HCC, Magnetic resonance imaging, Diffusion weighted imaging, Histologic grade

\section{Background}

Hepatocellular carcinoma (HCC) is the most common primitive hepatic cancer $[1,2]$. Imaging surveillance is a widely established tool that increases the probability of early detection of HCC, which is mandatory on patient at risk for this tumor since the treatment of $\mathrm{HCC}$ is different to other hepatic lesions [1]. According to the guidelines of National Comprehensive Cancer Network (NCCN) [3] and of European Association for the Study of the Liver (EASL) and American Association for the Study Liver Diseases National Comprehensive Cancer Network (AASLD), during the phase of $\mathrm{HCC}$ characterization, the diagnostic criteria should be used only for cirrhotic patients [4]. However, the up-to-date imaging-based criteria have several limits, counting the absence of recognized agreement concerning the precise descriptions of imaging features, binary classification (either definite or not definite HCC),

\footnotetext{
* Correspondence: r.fusco@istitutotumori.na.it

${ }^{1}$ Radiology Division, Istituto Nazionale Tumori IRCCS Fondazione G. Pascale -

IRCCS di Napoli, via Mariano Semmola, I-80131 Naples, Italy

${ }^{2}$ Hepatobiliary Surgical Oncology Division, Istituto Nazionale Tumori IRCCS

Fondazione G. Pascale - IRCCS di Napoli, via Mariano Semmola, I-80131 Naples, Italy
}

and disappointment to report non-HCC malignancies and vascular involvement [5]. Therefore, the American College of Radiology (ACR) has encouraged the use of Liver Imaging Reporting and Data System (LI-RADS) for the reading, recording and data collection of HCC nodules [6, 7]. Although imaging techniques allow identifying and characterizing of liver nodules with a higher diagnostic accuracy and Magnetic Resonance Imaging (MRI) is the diagnostic tool that should be chosen to survive HCC patients [8-10], however the gold standard to characterize liver lesions is still biopsy [11]. In fact, until now, histological analysis is the unique technique that allow to identify the histologic grade of $\mathrm{HCC}$, that is one of the most predictive factors of survival for HCC patients [11]. During the last years, the possibility to obtain functional data by Diffusion-weighted imaging (DWI), it has seen born a great interest on this technique. DWI has been applied to liver imaging as an excellent tool for detection and characterization of focal liver lesions, increasing clinical confidence and decreasing false positives [11-14]. Oncology is a major field of application of DWI. The analysis of DW images can be done qualitatively and quantitatively, through the apparent diffusion coefficient (ADC) map. 
Eco Planar Imaging (EPI) sequences are widely used for DWI, which are basically T2-W sequences, acquired with single shot technique and FS. Different series of DW images are acquired through modification of the gradient strength and magnitude, referred as b-value. One series should be obtained with a b-value of 0 , meaning no gradient is applied and consequently no diffusion information is retrieved, giving similar information as T2 FS sequences. Another series should be obtained with a low b-value $(\mathrm{b}<$ 100), for lesion detection, while series obtained with a high $\mathrm{b}$-value (such as $\mathrm{b}=800$ ) are important for liver lesion characterization [11-14]. DWI signal depends on the water mobility that is related to tissue characteristics [11]. Diffusion is quantified by a diffusion coefficient, the ADC. The ADC map is the graphical representation of the ratio of DW signal intensities and its measurements may discriminate between benign and malignant lesions. The ADC measurements are correlated to the sequence acquisition protocol and suffer from a lack of reproducibility, especially in respiratory triggering techniques, nodules of left liver lobe, smaller size and lesion heterogeneity [11]. Accurate estimation of $\mathrm{ADC}$ can be improved by acquiring a large number of b-values. ADC low values mean restricted diffusion, high $A D C$ values mean free or unimpeded diffusion. Malignant tissue shows signal hyperintensity in DWI and signal hypointensity in the apparent diffusion coefficient (ADC) map [11]. Several researchers investigated, therefore, the values of ADC for lesion characterization. The ADC values for malignant lesions vary in literature widely and show a significant overlap with benign and other malignant lesions [1114]. Le Bihan et al. as a first assessed the intravoxel incoherent motion (IVIM) and evaluated a more sophisticated method to define the relationship between signal attenuation and increasing $b$ value that separately reproduce tissue diffusivity and tissue perfusion $[12,13]$. IVIM data can be assessed qualitatively and quantitatively. The lesion characterization is done easier by quantitative data, while qualitatively method helped the detection of nodule [13]. In clinical practice DWI is widely employed after neoadjuvant therapy [14] or ablative techniques [15], to assess the efficacy of treatment. An emerging field of application of DWI is the evaluation of histological grade of the tumor [16]. Several researches have assessed the relationship between functional parameters obtained by DWI and histological grade of HCC [16-19]. Considering that the histologic grade of $\mathrm{HCC}$ is one of the most predictive factors of reappearance and survival after treatment and transplantation [16-19], the probability that imaging analysis could be associated to the histologic grade to selecting the therapeutic approach should guide the proper treatment of patient.
Our purpose is reporting an overview and update of the role of DWI in assessment of histologic grade of HCC.

\section{Methods}

This overview and update is the result of autonomous studies without protocol and registration number.

\section{Search criterion}

We evaluated several electronic databases, PubMed (US National Library of Medicine, http://www.ncbi.nlm.nih.gov/pubmed), Scopus (Elsevier, http://www.scopus.com/ ), Web of Science (Thomson Reuters, http://apps.webofknowledge.com/) and Google Scholar (https://scholar.google.it/), using as search criteria the following key words: "hepatocellular carcinoma" AND "diffusion magnetic resonance imaging" AND "histologic grade", "hepatocellular carcinoma" AND "intravoxel incoherent motion" AND "histologic grade", "hepatocellular carcinoma" AND "multimodal imaging" AND "histologic grade". Our analysis enclosed the time between January 2000 and October 2017. Also, we evaluated the references of the searched studies for documents not indexed in the electronic databases. We retained solely the papers recording DWI results in the evaluation of histologic grade of HCC. Articles published in the English language from January 2000 to October 2017 were included. The absence of full text, overview analysis and conference papers were considered as exclusion criteria.

\section{Histological grading assessment in HCC}

The classical and most commonly adopted grading system for HCC is Edmondson-Steiner (ES) [20], published in the 1954 that organized the tumors in 4-tier histological grade distribution (Table 1). In contrast, and most likely due to differences from the ES classification, the World Health Organization (WHO) classification organized tumors in 3-tiers (Table 1) [21]. Usually the researches, when WHO classification is adopted, tend to assess each grade individually $(\mathrm{G} 1 \times \mathrm{G} 2 \times \mathrm{G} 3)$, while when is adopted ES classification, they dichotomize them in low $(\mathrm{G} 1+\mathrm{G} 2)$ and high grades $(\mathrm{G} 3+\mathrm{G} 4)$.

\section{Results}

We collected 170 studies from the literature research from January 2000 to October 2017 considering the key words described above. However, 132 papers have different topic respect to correlation between HCC histologic grade and DWI and 24 studies corresponded to more than one excluded criteria. Therefore, fourteen articles were included at the end (Fig. 1). 
Table 1 Histological features according to Edmondson and Steiner (ES) and WHO classification

\begin{tabular}{|c|c|c|c|c|}
\hline Classification & Grades & Architecture & Cytology & Other features \\
\hline \multirow[t]{4}{*}{$\begin{array}{l}\text { Edmondson } \\
\text { and Steiner }\end{array}$} & I & - & - & $\begin{array}{l}\text { Areas of carcinoma where } \\
\text { distinction from hyperplastic } \\
\text { liver is difficult }\end{array}$ \\
\hline & $\|$ & $\begin{array}{l}\text { Trabecular, frequent acini (lumen } \\
\text { varying from tiny canaliculi to } \\
\text { large thyroid-like spaces) }\end{array}$ & $\begin{array}{l}\text { Resemblance to normal hepatic cells; larger } \\
\text { nuclei; abundant acidophilic cytoplasm }\end{array}$ & $\begin{array}{l}\text { Cell borders sharp and clear cut; } \\
\text { acini containing bile or protein } \\
\text { precipitate }\end{array}$ \\
\hline & III & $\begin{array}{l}\text { Distortion of trabecular structure, } \\
\text { acini less frequent than grade II }\end{array}$ & $\begin{array}{l}\text { Larger, more hyperchromatic nuclei, } \\
\text { granular but less acidophilic cytoplasm }\end{array}$ & $\begin{array}{l}\text { Acini are less frequent; tumor giant } \\
\text { cells may be numerous }\end{array}$ \\
\hline & IV & $\begin{array}{l}\text { Medullary, less trabeculae, rare } \\
\text { acini }\end{array}$ & $\begin{array}{l}\text { Highly hyperchromatic nuclei, scanty } \\
\text { cytoplasm, with fewer granules }\end{array}$ & $\begin{array}{l}\text { Loss of cell cohesiveness; giant, } \\
\text { spindle or short-plump cells can } \\
\text { be found }\end{array}$ \\
\hline \multirow[t]{4}{*}{$\begin{array}{l}\text { World Health } \\
\text { Organization }\end{array}$} & $\begin{array}{l}\text { Well } \\
\text { differentiated }\end{array}$ & $\begin{array}{l}\text { Thin trabecular, frequent acinar } \\
\text { structures }\end{array}$ & Minimal atypia & Fatty change is frequent \\
\hline & $\begin{array}{l}\text { Moderately } \\
\text { differentiated }\end{array}$ & $\begin{array}{l}\text { Trabecular ( } 3 \text { or more cells in } \\
\text { thickness) and acinar }\end{array}$ & $\begin{array}{l}\text { Abundant eosinophilic cytoplasm, round } \\
\text { nuclei with distinct nucleoli }\end{array}$ & $\begin{array}{l}\text { Bile or proteinaceous fluid within } \\
\text { acini }\end{array}$ \\
\hline & $\begin{array}{l}\text { Poorly } \\
\text { differentiated }\end{array}$ & Solid & Moderate to marked pleomorphism & $\begin{array}{l}\text { Absence of sinusoid-like blood } \\
\text { spaces }\end{array}$ \\
\hline & Undifferentiated & Solid & $\begin{array}{l}\text { Little cytoplasm, spindle, or round-shaped } \\
\text { cells }\end{array}$ & - \\
\hline
\end{tabular}

\section{Discussion}

The accurate detection of histologic grade of $\mathrm{HCC}$ is thought a main parameter in planning of the therapeutic approach [22]. Seeing that histological analysis of small doubtful nodule is often not feasible due to their location, the role of pre-operative imaging for the assessment of well, moderate and poorly differentiated HCCs is crucial [23]. DWI is a functional MRI technique that allows quantitative evaluation of water proton diffusion in tissues. HCC is characterized by increased cellularity and, thus, have restricted diffusion [11]. Intravoxel incoherent motion (IVIM) is a recently developed DWI-derived tool. IVIM can separate the effects of perfusion-related diffusion from pure molecular diffusion [11]. DWI and IVIM enable improved detection and characterization of $\mathrm{HCC}$
[11]. According to Granata et al. [11] DWI and IVIM should be a role in predicting of the histological grade of HCC. In fact, they showed a good correlation between ADC, fp (perfusion fraction), and Dt (tissue diffusivity) and tumoral grading. ROC analyses showed that an ADC value of $2.11 \times 10-3 \mathrm{~mm} 2 / \mathrm{sec}$, an $\mathrm{fp}$ value of $47,33 \%$ and an Dt value of $0.94 \times 10-3 \mathrm{~mm} 2 / \mathrm{sec}$ were the most accurate cut off levels to discriminate high grade versus low grade, with a sensitivity and specificity for ADC of 100 and $100 \%$, for fp of 100 and $89 \%$, for Dt of 100 and $74 \%$, respectively. Guo et al. assessed the relationships of signal intensity (SI) and ADC with the histological grade in 27 resected HCC patients. They showed that there were no significant differences in ADC parameters or SI between higher or lower grade of HCC nodules. In fact the overall

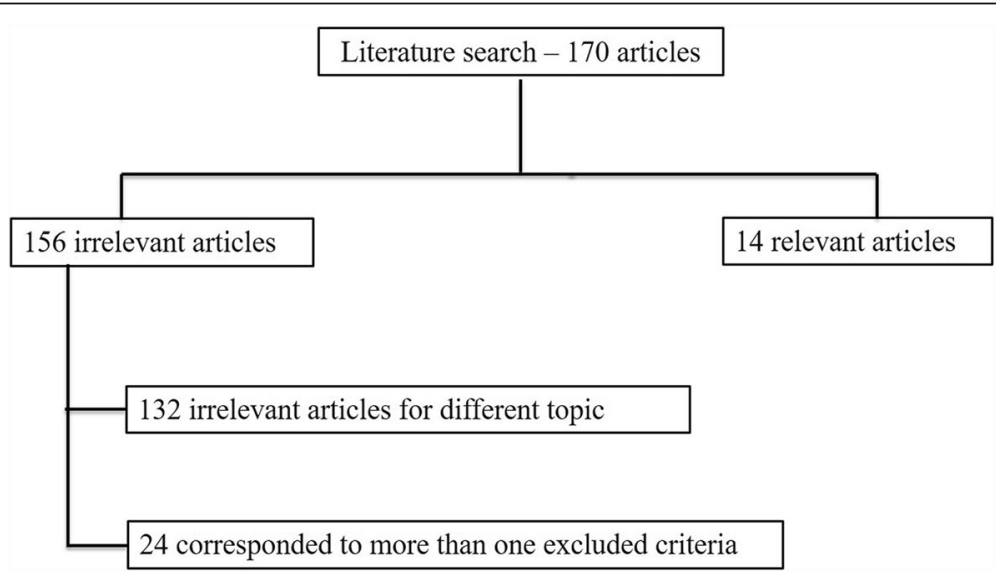

Fig. 1 Included and excluded studies in systematic review 
ADC rate for all cases was $1.28 \pm 0.19 \times 10-3 \mathrm{~mm} 2 / \mathrm{s}$. The ADC was $1.16 \pm 0.16 \times 10-3 \mathrm{~mm} 2 / \mathrm{s}$ for poorly differentiated nodules, lower than the well $[1.43 \pm 0.09 \times 10$ $3 \mathrm{~mm} 2 / \mathrm{s}]$ and moderately $[1.34 \pm 0.19 \times 10-3 \mathrm{~mm} 2 / \mathrm{s}]$ differentiated HCCs. The overall SI value was $75.66 \pm 32.94$. The mean SI value for the moderately differentiated HCCs was $54.37 \pm 28.37$, lower than the well $(90.78 \pm 27.49)$ and poorly $(86.77 \pm 31.51)$ differentiated [16]. Nakanishi et al. showed not only the utility of DWI for histological tumor grading, but also that ADC should be used as a preoperative prediction of early recurrence [22]. DWI is a valuable diagnostic tool, that allows not invasively characterizing biological tissues by measurement of properties of water diffusion, however there are results which are in contrast each one [12-19, 24-30]. Chen et al., in a meta-analysis, found that for differentiating well differentiated lesions from higher grades, DWI showed a low sensitivity (54\%), high specificity (90\%), and an excellent diagnostic performance (area under curve $(\mathrm{AUC})=0.9311$ ). Conversely, in differentiating poorly differentiated lesion from lower grades, the sensitivity was $84 \%$, the specificity $48 \%$, showing a moderately high diagnostic performance [18]. Nasu et al. evaluated 125 resected HCCs showing no association between histological grade and ADC, while they found that SI of the HCC increased in higher grade [23]. Instead, Muhi et al. found significant changes in SI and ADC between different grades of $98 \mathrm{HCC}$ nodules, although there was still considerable overlapping [24]. Nishie et al. found a relationship between ADC parameters and HCC histological data, but the difference was significant only between well-differentiated and poorly differentiated lesions [25]. Recent technique advance has endorsed the application of IVIM in predicting the histological grade of HCC [17]. By using the IVIM model diffusion features can be disconnected from pseudo diffusion caused by perfusion [12, 13]. According to Woo et al. IVIM-derived diffusion values (diffusion coefficient, Dt) had considerably higher diagnostic performance compared to ADC in discerning high grade (Fig. 2) from low grade HCC (Fig. 3) [17]. Conversely Granata et al. showed that the ADC had the best diagnostic performance, in comparison of $\mathrm{fp}$ and Dt [11].
However the mayor limit of DWI and IVIM parameters to discriminate the histological grade of HCC, as suggested by Ichikawa et al., is depending on the fitting methods used to obtained functional parameters, thus the fitting would be robust even though some errors might have occurred during image acquisition [29]. A prospective study with a larger cohort would be necessary to confirm the usefulness of the IVIM parameters for distinguishing poorly differentiated HCCs from other HCC grades and to establish the advantages of this method [29].

Several tumor features evaluated by imaging techniques can be associated with HCC prognosis after treatment [31]. Microvascular invasion (MVI), defined as microscopically detected tumor thrombi within small tumor or peritumoral vessels, to day, is considered a major risk factor of recurrence [31]. DWI and DWI-based approaches (IVIM and Kurtosis) play a pivotal role in assessment of MVI. Several researches have shown that higher tumor-to-liver signal intensity ratio and lower ADCs value can predict MVI [32-34]. This could be due to higher cellularity with restricted diffusion and decreased perfusion in MVI HCCs compared with no MVI HCC. Wang et al. assessed the role of kurtosis in HCC showing that mean kurtosis values increased in MVI positive patients so that those can be independent risk factors for MVI [35].

Another field of attention is the role of DWI in the assessment of immunotherapy. To date at the best of our knowledge there are not present in literature studies that describe the role of DWI in the assessment of HCC response after immunotherapy. However, Qin et al. [36] report the promising results of DWI in glioblastoma patients subjected to anti-PD1 therapy in order to differentiate patients who derive therapeutic benefit from those who do not. Preliminary data from advanced MRI assessment suggests that increase in volume of abnormal tissue with contrast enhancement, edema, and intermediate ADC occurs in most patients during the initial months of anti-PD1 \pm anti-CTLA-4 immunotherapy. Among patients who appear to achieve therapeutic benefit, subsequent improvement in these MRI markers

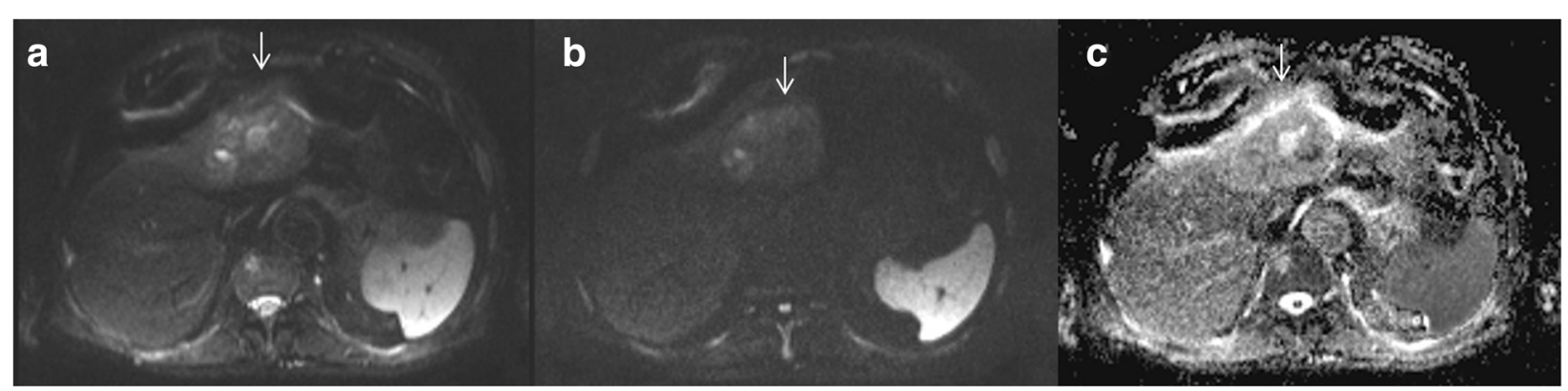

Fig. 2 High-grade HCC. DWl sequences: in A b50 s/mm²; in B b800 s/mm2 and in C ADC map 


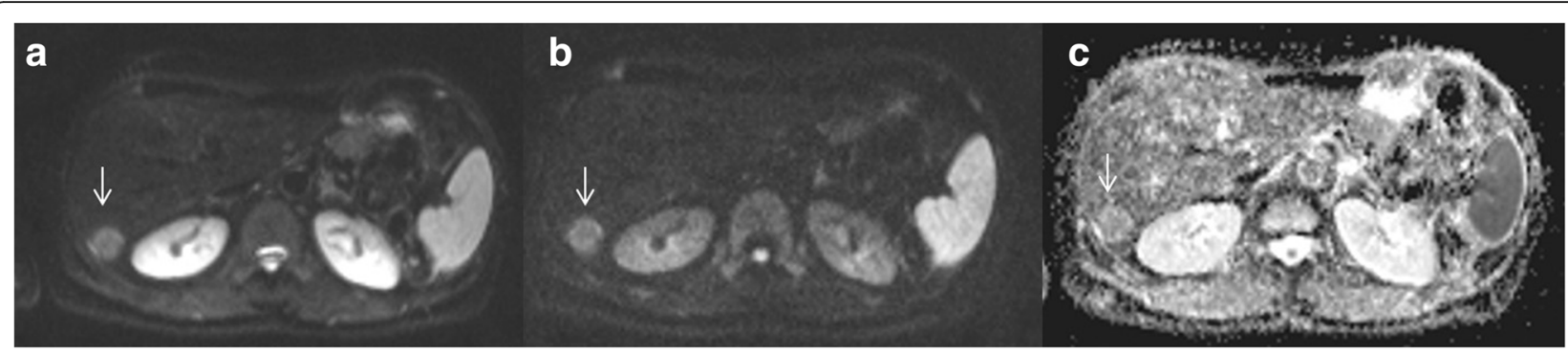

Fig. 3 Low-grade HCC. DWI sequences: in A b50 s/mm²; in B b800 s/mm2 and in C ADC map

was observed. Their findings suggest that volumetric change in ADC may correlate better with therapeutic benefit than RANO criteria measures. Therefore, future endpoint could be the evaluation of HCC tissue and in particular of immune cell infiltrate using DWI after immunotherapy [36].

Although there are the great advantages due to DWI and DWI-based approaches in detection and characterization of HCC, and DWI has been included in the Liver Imaging Reporting and Data System [9, 10], these approaches have several limitations. First, the performance of DWI for detection could be degraded due to the not standardized acquisition protocol, including the determination of optimal $b$ values and breathing techniques across different modalities and medical centers. Therefore, universal thresholds for $\mathrm{ADC}$ and other quantitative parameters may not be acquirable. Second, DWI is sensitive to motion artifact; thus, detection and characterization of lesions can be mostly affected in the presence of motion artifacts [29].

\section{Conclusion}

The histologic grade of HCC is one of the most prognostic features of reappearance and survival after surgical treatment and transplantation. The probability that the imaging could identify the histologic grade of HCC should be a useful tool to guide the patient management. In this context, MRI study with the DWI sequences should be the method to choose because the ADC and IVIM-derived parameters are related to the histological grade of HCC. However, a larger study group would be necessary to confirm the usefulness of the IVIM parameters for distinguishing poorly differentiated HCCs from other HCC grades.

\footnotetext{
Abbreviations

AASLD: Association for the Study Liver Diseases National Comprehensive Cancer Network; ACR: American College of Radiology; ADC: Apparent diffusion Coefficient; AUC: Area under curve; Dt: Tissue diffusivity; DWI: Diffusion-weighted imaging; EASL: European Association for the Study of the Liver; fp: Perfusion fraction; HCC: Hepatocellular carcinoma; IVIM: Intravoxel incoherent motion; LI-RADS: Liver Imaging Reporting and Data System; MRI: Magnetic Resonance Imaging; NCCN: National Comprehensive Cancer Network
}

\section{Acknowledgements}

The authors are grateful to Alessandra Trocino, librarian at the National Cancer Institute of Naples, Italy. Additionally, authors are grateful to Assunta Zazzaro and Rita Guarino for collaboration.

\section{Availability of data and materials}

Data sharing not applicable to this article as no datasets were generated or analysed during the current study.

\section{Authors' contributions}

VG conceived of the study, and participated in its design, coordination and drafting of the manuscript. RF participated in the studies collection and drafted the manuscript. SF, OC, MP, RP, FI, AP participated in the studies collection. All authors read and approved the final manuscript. Guarantor of the manuscript is Vincenza Granata. Each author have participated sufficiently in any submission to take public responsibility for its content.

Ethics approval and consent to participate

Not applicable.

\section{Consent for publication}

Not applicable.

\section{Competing interests}

The authors declare that they have no competing interests.

\section{Publisher's Note}

Springer Nature remains neutral with regard to jurisdictional claims in published maps and institutional affiliations.

Received: 28 May 2018 Accepted: 22 June 2018

Published online: 03 July 2018

\section{References}

1. Bruix J, Sherman M. Management of hepatocellular carcinoma: an update. Hepatology. 2011;53:1020-2.

2. Piccirillo M, Granata V, Albino V, Palaia R, Setola SV, Petrillo A, Tatangelo F, Botti G, Foggia M, Izzo F. Can hepatocellular carcinoma (HCC) produce unconventional metastases? Four cases of extrahepatic HCC Tumori. 2013; 99(1):e19-23.

3. NCCN Clinical Practice Guidelines in Oncology on hepatobiliary Cancer Version 2016. http://www.nccn.org.

4. European Association for Study of Liver; European Organisation for Research and Treatment of Cancer. EASL-EORTC clinical practice guidelines: management of hepatocellular carcinoma. Eur J Cancer 2012;48(5):599-641. https://doi.org/10.1016/j.ejca.2011.12.021. Erratum in: Eur J Cancer 2012; 48(8):1255-1256.

5. An C, Rakhmonova G, Choi JY, et al. Liver imaging reporting and data system (LI-RADS) version 2014: understanding and application of the diagnostic algorithm. Clin Mol Hepatol. 2016;22(2):296-307.

6. American College of Radiology Liver Imaging Reporting and Data System Version 2014. ACR Web site http://www.acr.org/Quality-Safety/Resources/ LIRADS. Accessed 15 Apr 2016.

7. Santillan CS, Tang A, Cruite I, Shah A, Sirlin CB. Understanding LI-RADS. a primer for practical use Magn Reson Imaging Clin N Am. 2014;22:337-52. 
8. Granata V, Petrillo M, Fusco R, Setola SV. de Lutio di Castelguidone E, Catalano O, Piccirillo M, albino V, Izzo F, Petrillo a. Surveillance of HCC patients after liver RFA: role of MRI with Hepatospecific contrast versus three-phase CT scan-experience of high volume oncologic institute. Gastroenterol Res Pract. 2013;2013:469097.

9. Granata V, Fusco R, Avallone A, Catalano O, Filice F, Leongito M, Palaia R, Izzo F, Petrillo A. Major and ancillary magnetic resonance features of LI-RADS to assess HCC: an overview and update. Infect Agent Cancer. 2017;12:23.

10. Granata V, Fusco R, Avallone A, Filice F, Tatangelo F, Piccirillo M, Grassi R, Izzo F, Petrillo A. Critical analysis of the major and ancillary imaging features of LI-RADS on 127 proven HCCs evaluated with functional and morphological MRI: lights and shadows. Oncotarget. 2017:8(31):51224-37.

11. Granata V, Fusco R, Catalano O, Guarino B, Granata F, Tatangelo F, Avallone A, Piccirillo M, Palaia R, Izzo F, Petrillo A. Intravoxel incoherent motion (IVIM) in diffusion-weighted imaging (DWI) for hepatocellular carcinoma: correlation with histologic grade. Oncotarget. 2016:7(48):79357-64.

12. Le Bihan D, Breton E, Lallemand D, Grenier P, Cabanis E, Laval-Jeantet M. MR imaging of intravoxel incoherent motions: application to diffusion and perfusion in neurologic disorders. Radiology. 1986;161:401-7.

13. Le Bihan D, Breton E, Lallemand D, Aubin ML, Vignaud J, Laval-Jeantet M. Separation of diffusion and perfusion in intravoxel incoherent motion MR imaging. Radiology. 1988;168:497-505.

14. Granata V, Fusco R, Catalano O, Filice S, Amato DM, Nasti G, Avallone A, Izzo F, Petrillo A. Early assessment of colorectal Cancer patients with liver metastases treated with Antiangiogenic drugs: the role of Intravoxel incoherent motion in diffusion-weighted imaging. PLoS One. 2015;10(11): e0142876.

15. Granata V, Fusco R, Catalano O, Piccirillo M, De Bellis M, Izzo F, Petrillo A. Percutaneous ablation therapy of hepatocellular carcinoma with irreversible electroporation: MRI findings. AJR Am J Roentgenol 2015;204(5):1000-7. d.

16. Guo W, Zhao S, Yang Y, Shao G. Histological grade of hepatocellular carcinoma predicted by quantitative diffusion-weighted imaging. Int J Clin Exp Med. 2015;8(3):4164-9.

17. Woo S, Lee JM, Yoon JH, Joo I, Han JK, Choi BI. Intravoxel incoherent motion diffusion-weighted MR imaging of hepatocellular carcinoma: correlation with enhancement degree and histologic grade. Radiology. 2014;270(3):758-67.

18. Chen J, Wu M, Liu R, Li S, Gao R, Song B. Preoperative evaluation of the histological grade of hepatocellular carcinoma with diffusion-weighted imaging: a meta-analysis. PLoS One. 2015;10(2):e0117661.

19. Li X, Li C, Wang R, Ren J, Yang J, Zhang Y. Combined application of Gadoxetic acid disodium-enhanced magnetic resonance imaging (MRI) and diffusion-weighted imaging (DWI) in the diagnosis of chronic liver disease-induced hepatocellular carcinoma: a meta-analysis. PLoS One. 2015;10(12):e0144247.

20. Edmondson HA, Steiner PE. Primary carcinoma of the liver: a study of 100 cases among 48,900 necropsies. Cancer. 1954;7:462-503.

21. World Health Organization Classification of Tumours by International Agency for Research on Cancer WHO classification of Tumours of the digestive system: volume 3. 4th revised ed. Lyon: International Agency for Research on Cancer; 2010.

22. Nakanishi M, Chuma M, Hige S, et al. Relationship between diffusion-weighted magnetic resonance imaging and histological tumor grading of hepatocellular carcinoma. Ann Surg Oncol. 2012;19(4):1302-9.

23. Nasu K, Kuroki Y, Tsukamoto T, et al. Diffusion-weighted imaging of surgically resected hepatocellular carcinoma: imaging characteristics and relationship among signal intensity, apparent diffusion coefficient, and histopathologic grade. AJR Am J Roentgenol. 2009;193:438-44.

24. Muhi A, Ichikawa T, Motosugi U, et al. High-b-value diffusion-weighted MR imaging of hepatocellular lesions: estimation of grade of malignancy of hepatocellular carcinoma. J Magn Reson Imaging. 2009;30:1005-11.

25. Nishie A, Tajima T, Asayama Y, et al. Diagnostic performance of apparent diffusion coefficient for predicting histological grade of hepatocellular carcinoma. Eur J Radiol. 2011;80:e29-33.

26. Zhou L, Rui JA, Wang SB, et al. Factors predictive for long-term survival of male patients with hepatocellular carcinoma after curative resection. J Surg Oncol. 2007;95(4):298-303.

27. Kim SH, Lim HK, Choi D, Lee WJ, Kim SH, Kim MJ, et al. Percutaneous radiofrequency ablation of hepatocellular carcinoma: effect of histologic grade on therapeutic results. AJR Am J Roentgenol. 2006;186:S327-33.
28. Ludwig JM, Juan C, Camacho JC, Nima Kokabi N, et al. The role of diffusionweighted imaging (DWI) in Locoregional therapy outcome prediction and response assessment for hepatocellular carcinoma (HCC): the new era of functional imaging biomarkers. Diagnostics (Basel). 2015;5(4):546-63.

29. Ichikawa S, Motosugi U, Hernando D, Morisaka H, Enomoto N, Matsuda M, Onishi H. Histological grading of hepatocellular carcinomas with Intravoxel incoherent motion diffusion-weighted imaging: inconsistent results depending on the fitting method. Magn Reson Med Sci. 2017;

30. Shan Q, Chen J, Zhang T, Yan R, Wu J, Shu Y, Kang Z, He B, Zhang Z, Wang J. Evaluating histologic differentiation of hepatitis $B$ virus-related hepatocellular carcinoma using intravoxel incoherent motion and AFP levels alone and in combination. Abdom Radiol (NY). 2017;42(8):2079-88.

31. Jiang HY, Chen J, Xia CC, Cao LK, Duan T, Song B. Noninvasive imaging of hepatocellular carcinoma: From diagnosis to prognosis. World J Gastroenterol. 2018;24(22):2348-62

32. Suh YJ, kim MJ, Jy C, Park MS, kim kW. Preoperative prediction of the microvascular invasion of hepatocellular carcinoma with diffusion-weighted imaging. Liver Transpl. 2012;18:1171-8.

33. Okamura S, Sumie S, Tonan T, Nakano M, Satani M, Shimose S, Shirono T, Iwamoto H, Aino H, Niizeki T, Tajiri N, Kuromatsu R, Okuda k NO, Torimura T. Diffusion-weighted magnetic resonance imaging predicts malignant potential in small hepatocellular carcinoma. Dig Liver Dis. 2016;48:945-52.

34. Xu P, Zeng M, Liu k S y, Xu C, Lin J. Microvascular invasion in small hepatocellular carcinoma: is it predictable with preoperative diffusionweighted imaging? J Gastroenterol Hepatol. 2014;29:330-6.

35. Wang WT, yang $L$, yang ZX, Hu XX, Ding y y X, Fu CX, Grimm R, Zeng MS, Rao SX. Assessment of microvascular invasion of hepatocellular carcinoma with diffusion kurtosis imaging. Radiology. 2018;286:571-80.

36. Qin L, Li X, Stroiney A, Qu J, Helgager J, Reardon DA, Young GS. Advanced MRI assessment to predict benefit of anti-programmed cell death 1 protein immunotherapy response in patients with recurrent glioblastoma. Neuroradiology. 2017;59(2):135-45.

\section{Ready to submit your research? Choose BMC and benefit from:}

- fast, convenient online submission

- thorough peer review by experienced researchers in your field

- rapid publication on acceptance

- support for research data, including large and complex data types

- gold Open Access which fosters wider collaboration and increased citations

- maximum visibility for your research: over $100 \mathrm{M}$ website views per year

At BMC, research is always in progress.

Learn more biomedcentral.com/submissions 